\title{
Acesso à Atenção Primária à Saúde em Curitiba: a percepção dos usuários que frequentam uma unidade de pronto atendimento (UPA).
}

Bruno Marach Bizinelli, Paulo Poli Neto, Guilherme Souza Cavalcanti de Albuquerque, Ramon

Eduardo Szymczak Conde, Felipe Calderon Scarin

\section{RESUMO}

Motivação: Evidências científicas apontam que um sistema fortemente baseado em Atenção Primária à Saúde (APS) promove melhores desfechos em saúde. No Brasil, a APS acontece, no sistema público, preferencialmente, no modelo de Estratégia de Saúde da Família. São considerados atributos essenciais da APS: o acesso, a longitudinalidade, a abrangência dos serviços e coordenação do cuidado. Esta pesquisa visou explorar as barreiras de acesso à APS na visão dos usuários que frequentam uma UPA (Unidade de Pronto Atendimento).

Método: Análise qualitativa baseada em entrevista semiestruturada com pacientes de uma Unidade de Pronto Atendimento de Curitiba, Paraná.

Resultados: Revelaram-se barreiras de acesso à APS: distância de casa, demora na resposta da APS, compreensão sobre o funcionamento do sistema, acesso facilitado à UPA para problemas não urgentes e horário de funcionamento abreviado da unidade básica de saúde (UBS).

Conclusões: Há percepção de que a UPA tem um acesso mais facilitado do que a UBS, apesar de não ser, em teoria, a porta de entrada oficial do sistema.

Palavras-chave: Atenção Primária à Saúde, Acesso aos Serviços de Saúde, Avaliação em Saúde, Pacientes.
Revista da Rede APS 2019

Publicada em: 17/12/2019

DOI:10.14295/aps.v1i3.39

Bruno Marach Bizinelli

(Médico de Família e Comunidade. Mestre em Saúde da Família. Professor da faculdade de Medicina das Faculdades Pequeno Príncipe (FPP))

\section{Paulo Poli Neto}

(Médico de Família e Comunidade. Professor doutor do departamento de Saúde Coletiva da UFPR)

Guilherme Souza Cavalcanti de Albuquerque

(Professor doutor do departamento de Saúde Coletiva da UFPR)

Ramon Eduardo Szymczak Conde (Acadêmico de Medicina da UFPR)

Felipe Calderon Scarin

(Acadêmico de Medicina da UFPR)

Correspondência para: Bruno Marach Bizinelli

bruno.bizinelli@hotmail.com 


\section{INTRODUÇÃO}

Desde o relatório de Dawson em 1920, a Atenção Primária à Saúde (APS) aparece com um papel central nos sistemas de saúde. As comparações entre sistemas de saúde têm demonstrado que sistemas orientados e coordenados pela APS costumam ser mais efetivos e equitativos. (STARFIELD, 2002; ALMA-ATA, 1978; CASTRO et al., 2012; O'DONNELL et al., 2016).

Starfield (2002) definiu a APS em quatro atributos essenciais. $O$ acesso ou primeiro contato por um problema de saúde novo ou novos contatos por um problema de saúde antigo. A longitudinalidade que compreende o seguimento presencial, virtual e dos registros eletrônicos. A integralidade ou abrangência que compreende a organização dos serviços ofertados de modo a lidar com as demandas em saúde mais prevalentes. $E$ a coordenação do cuidado, papel da APS ao se relacionar com os demais serviços de acordo com a necessidade das pessoas sob sua responsabilidade. (STARFIELD, 2002; CASTRO et al., 2012; TESSER; NORMAN; VIDAL, 2018).

$\mathrm{O}$ acesso adequado permite que o paciente tenha sua demanda de saúde sanada em tempo hábil pela equipe que o conhece. Cabe também à APS evitar que as situações mais prevalentes cheguem a serviços muito especializados ou aos prontosocorros. (CASTRO et al., 2012; TESSER; NORMAN; VIDAL, 2018; MURRAY; BERWICK, 2003; CAMPOS, 2014).

Um dos grandes desafios da APS, é a organização do acesso e das agendas dos profissionais de saúde. Murray (2003) chama atenção para que a realidade da APS muitas vezes compreende um embate entre pacientes e recepcionistas sobre como conseguir um horário na agenda do médico. Aponta grande possibilidade de melhoria mudando o processo de trabalho. (TESSER; NORMAN; VIDAL, 2018; MURRAY; BERWICK, 2003; MENDES, 2016).

Um dos fatores chaves é a quantidade de pessoas por equipe ou médico. O número de 2 mil a 2.300 pacientes por equipe (considerando faixa etária ou comorbidades) parece ser o mais adequado para oferecer um acesso facilitado. (ROCHA; BOCCHI; GODOY, 2016).
Uma outra questão é o tamanho da equipe, na maioria dos países com APS forte, há dois ou três profissionais de saúde para cada 2 a 2,5 mil pessoas. Na estratégia de saúde da família (ESF), recomendase uma população de 3,5 mil pessoas para uma equipe com cerca de 9 profissionais. (ROCHA; BOCCHI; GODOY, 2016).

No Brasil, existem também as Unidades de ProntoAtendimento (UPA's), que acabam exercendo papel de porta de entrada. Elas são consideradas unidades intermediárias, entre as unidades de saúde (UBS) e os hospitais. (OLIVEIRA; ARAUJO; GARCIA, 2018; OLIVEIRA et al., 2015).

Uma das dificuldades dessas portas de entrada nãohospitalares de urgência é a definição do seu papel, já que não deveriam oferecer a função de continuidade da APS, tampouco a resolutividade dos hospitais para os casos mais graves. Estudos brasileiros apontam que mais de $90 \%$ das situações clínicas atendidas nas UPAs poderiam ser atendidas pela APS. (MURRAY; BERWICK, 2003; PICOLI; CAZOLA; MAURER, 2016).

O objetivo da pesquisa, portanto, é o de compreender a percepção dos usuários sobre as diferenças de acesso a uma UPA em relação a sua UBS de referência.

\section{Metodologia}

$A$ pesquisa foi realizada em Curitiba-PR, que conta com 110 UBS e 10 UPAs. A escolha da UPA a ser pesquisada foi definida em função do distrito com a melhor adequação entre população usuária do SUS e profissionais de saúde das UBS. Para isso, foram utilizados os critérios de população por equipe de ESF, taxa de frequentação (número de consultas por ano em média por habitante) e extensão de uso. Considerou-se também o conceito de pressão assistencial (número de consultas por período dividido pelos dias trabalhados neste período). Esses dados foram obtidos a partir do Boletim de Informação e Monitoramento da APS da Secretaria Municipal da Saúde de Curitiba e cedidos pela mesma instituição. Do distrito estudado, 4 das 6 unidades, modelo ESF, tinham, no máximo 3.500 habitantes por equipe. (ROCHA; BOCCHI; GODOY, 2016; GUSSO; FAORO et al., 2015, BRASIL, 2017). 


\section{APS em Revista}

Vol. 1, n. 3, p. 198/205 | Setembro/Dezembro - 2019

ISSN 2596-3317 - DOI 10.14295/aps.v1i3.39

Bizinelli, B. M.; Poli Neto, P.; Albuquerque, G. S. C.; Conde, R. E. S.; Scarin, F. C.

Os participantes da pesquisa selecionados foram aqueles classificados como os de menor urgência de acordo com o protocolo de Manchester. (DISTRITO FEDERAL, 2017). Todos os pesquisados foram abordados em um horário em que a sua unidade de saúde de referência estivesse aberta. Todos os usuários consentiram com o Termo de Consentimento Livre e Esclarecido. Foram excluídas gestantes, pacientes abaixo de 18 anos, analfabetos e os que estivessem em urgências ou emergências.

A entrevista semiestruturada baseou-se em duas perguntas disparadoras: "Quais os motivos de ter procurado a UPA para seu atendimento hoje? " e "Você acha que poderia ter procurado a sua unidade de saúde para resolver este problema?".

Foi usado o mecanismo de saturação de dados para determinar a suspensão das entrevistas e depois geradas categorias para análise dos dados. As entrevistas foram áudio-gravadas e transcritas separadamente.-_(FONTANELLA et al., 2011).

A análise dos dados foi realizada pela técnica de análise de conteúdo proposta por Bardin. (MINAYO, 2014).

O projeto de pesquisa foi aprovado no Comitê de Ética em pesquisa da NN [eliminado para efeitos da revisão por pares], sob o CAAE 80185817.1.0000.0102.

\section{RESUltAdOS E DiscuSSÃO}

Foram realizadas 13 entrevistas. Considerou-se a saturação de dados para duas categorias: "Acesso facilitado na UPA" e "Acesso facilitado para grupos ou problemas populacionais específicos".

\section{Acesso facilitado na UPA}

Como Starfield (2002) salienta, a partir de um sintoma ou medo do que possa representá-lo, as pessoas vão buscar a resposta conhecida mais rápida ou prática. A maioria das pessoas pode não compreender, sozinhas, a gravidade de seu quadro. (STARFIELD, 2002; TESSER, 2017).

O entrevistado (E) 3 falou a respeito:
“Pesquisador $(P)$ : Quando você sente alguma coisa, onde você vai?

E3: É aqui.

(P): Você já tentou ir à UBS?

E3: Não.

(P): Por que que você prefere vir aqui?

E3: Porque é mais perto da minha casa e é mais vazio."

Estudos demonstram que há maior utilização da UBS quando há proximidade geográfica. Maiores distâncias estão associadas a piores desfechos em saúde, aumento de hospitalizações e iniquidade elevada. (STARFIELD, 2002; TESSER; NORMAN; VIDAL, 2018; RAMOS; HOFFMANN; SPRECKELSEN, 2018).

Normalmente, pensamos no acesso como um fator específico relacionado à oferta e à demanda. Porém, outros fatores individuais também influenciam: como preferências, gostos e informações. A falta de um ponto de entrada acessível pode não permitir que a orientação indique o cuidado mais apropriado. (STARFIELD, 2002; MENDES, 2016).

Vejamos o próximo trecho:

“(P): Você acha que poderia ter procurado a Unidade por causa desse problema [...]?

E1: Já procurei. Está agendado só pro dia 18. Lá é meio complicado assim [...] Você tem que ir bem cedo. Umas 5 horas da manhã pra você conseguir. E às vezes você não consegue. Não pega a vaga".

Neste trecho percebe-se que apesar da tentativa de acesso à UBS, a consulta foi marcada para 13 dias após o contato, o entrevistado procurou a UPA para resolução.

Outro trecho:

“E11: A gente vai na unidade de madrugada e dependendo do lugar que a gente mora a gente não tem condições [...] que a gente pode ser assaltado [...] Aí a gente vem direto aqui já. 
Uns 10 anos atrás eu ia direto na Unidade, mas o atendimento lá não tá muito bom. Porque eles fazem a gente esperar. A gente procura a UBS porque a gente tá com dor, tá com algum problema, e eles demoram pra identificar o problema".

A dificuldade de acesso mostra que enquanto na UPA não há limite sobre o número de atendimentos, na UBS há. O tempo de espera na UBS pode ser maior, gerando filas, como aparece na fala do E2:

“E2: É, sempre primeiro a gente vai lá (na unidade), meio fora assim da linha [...] que passa o ônibus. Daí a gente pega e vem aqui então, que tá sabendo que lá já passou do horário da senha, entendeu?

(P): Mas a unidade é mais perto daqui da casa da senhora ou mais longe daqui?

E2: É, vamos supor que é a mesma distância. Sempre eles pedem mais é [...] lá por sete horas (hora de chegada), entendeu? Mas essa sete horas [...] já tem que ir bem antes das sete horas pra formar fila lá fora pra quando já abrir o posto, daí já conseguir a consulta”.

Starfield (2002) destaca que um acesso pior leva à dificuldade de outros atributos essenciais. Outro aspecto é o horário de funcionamento, enquanto a UPA funciona 24 horas por dia, 7 dias da semana, a UBS costuma ter um horário menor (8 a 12 horas/dia). (STARFIELD, 2002).

“(P): Você acha que você poderia ter procurado a tua UBS pra resolver esse problema?

E7: Ah, não sei. Provavelmente o atendimento seria bem mais demorado. Porque lá não é um 24 horas. Se fosse 24 horas eu poderia ter ido ontem já. Que ontem eu já tava com a coluna ruim. Só não vim aqui porque era mais distante".

Alguns autores descreveram a importância de um horário mais estendido de atendimento na APS, resultando em diminuição na pressão assistencial em hospitais e de internamentos. (SALISBURY, 2002; FORD, 2018).
A dificuldade de acesso por conta do horário de funcionamento também é descrita em outros países. O NHS (National Health Service), na Inglaterra, descreveu o horário das cooperativas de General Practioners (GP's), correspondentes aos médicos de família no Brasil, sendo conformado como uma barreira de acesso. (GODDARD, 2008).

Murray (2003) descreve que pelo menos uma a cada quatro pessoas não conseguem acesso oportuno à APS. O autor defendeu uma forma de acesso ampliada, chamada acesso avançado. Propunha fundamentalmente uma reorganização do serviço e equipes para melhoria do acesso. (MURRAY; BERWICK, 2003; SUBRAMANIAN, 2009).

Em experiências internacionais com APS avaliadas como forte, não há uma estrutura semelhante às UPAs. Cabe às unidades de APS a oferta de atendimento nos horários não comerciais. (BARBOSA; ELIZEU; PENNA, 2013; BERCHET; NADER, 2016).

\section{Acesso facilitado para grupos ou problemas populacionais específicos}

E4: [...] eu tive que ir (na UBS) por causa que eles me fizeram o pedido da ecografia do abdômen (na UPA). Então eu tinha que passa pelo médico pra daí eu conseguir fazer a ecografia. E quando eu venho aqui (UPA) normalmente é só quando me ataca a dor mesmo.

Neste trecho, a UPA detectou um problema pediu um exame e o paciente necessitou ir à UBS para esta validação, ou seja, gerando dois custos para resolução de um problema.

Já na fala do E5:

“(P): Quais que foram os motivos, pra você ter procurado a UPA para o teu atendimento?

E5: Hoje foi por causa que minha garganta, tá inflamada.

$(P)$ : E você acha que você poderia ter procurado a Unidade para resolver esse problema?

E5: Ali onde eu moro o postinho de saúde não atende esse tipo de doença. [...] É que ali para você conseguir um atendimento você 
tem que marcar na hora ali e ir só no outro dia ali.

Para este entrevistado, há um "tipo de doença" que pode ser atendido pela UBS, e não era o seu caso. Assim como na fala do E4, o problema poderia ser resolvido pela unidade, porém não foi. Na fala do E5, parece que outros problemas seriam mais relacionados ao papel da UBS, não o seu.

O E9 pode complementar essa discussão:

(P): Você procura a UBS em algum momento?

E9: Ah... Lá é difícil de você conseguir consulta! É muito raro!

É só emergência, idoso ou gestante.

$(P)$ : Mas você chega a ir lá primeiro, ou você vem aqui direto?

E9: Lá primeiro, pra depois vim pra cá... Eles ... da avaliação [...] eles mandam pra cá."

Muitas vezes a APS faz opções de atendimentos, por questões relacionadas à gestão ou às crenças da própria equipe. Um dos exemplos citadas pelo entrevistado: a agenda voltada para atendimentos específicos (gestantes e idosos).

O debate entre o agendamento para o dia, ou no máximo para o dia seguinte e o agendamento preferencial para determinados problemas ou situações clínicas (programas) é frequente na APS brasileira. (SUBRAMANIAN, 2009).

Assim, o acesso é o resultado da combinação de vários fatores como localização, custo e adequação de serviços, perfil epidemiológico e nesta situação a disponibilidade de recursos. É possível que o acesso restrito na APS seja decorrente de um modelo sobrecarregado, gerando demandas duplas (para a UPA e para a APS) como na fala do E4. (TESSER; NORMAN; VIDAL, 2018).

Eugênio Vilaça Mendes (2016) traz a importância de que o sistema de saúde seja organizado em uma economia de escala. Com objetivo de trazer os melhores resultados possíveis, controlando os recursos disponíveis. Assim, repensar o papel de cada equipamento de saúde, levando em conta o seu custo e redimensionando o seu papel parece ser fundamental para sua conformação. (MENDES, 2016).

O limite entre otimização de recursos e adequação das equipes é difícil de ser atingido. É claro que a solução à sobrecarga de trabalho em um modelo constantemente sobrecarregado vai muito além de contratação de profissionais, devendo levar em conta as relações dentro do sistema. (MENDES, 2016; CARRAPATO et al., 2016).

A capacidade de resposta da APS poderia ser ampliada para diminuir os encaminhamentos à UPA por falta de acesso. Uma das formas de resolução apontadas por Tesser, Norman e Vidal (2018) seria a ampliação da capacidade clínica dos enfermeiros, aumentando a abrangência dos serviços destes profissionais. (TESSER; NORMAN; VIDAL, 2018; BARBOSA; ELIZEU; PENNA, 2013).

Quando se opta por atender um paciente porque o prioriza na agenda, frente a um contexto de sobrecarga das equipes, tira-se a possibilidade de que alguém com maior necessidade tenha acesso ao serviço, procurando outra forma de resolver seu problema. Muitas vezes essas escolhas são feitas baseadas em opiniões e não em evidências científicas. (TESSER; NORMAN; VIDAL, 2018; MURRAY; BERWICK, 2003; OLIVEIRA et al., 2015).

\section{CONSIDERAÇÕES FINAIS}

Os usuários entrevistados moravam em regiões pertencentes a UBS com uma relação população/profissionais de saúde considerada adequada pela política nacional, ainda assim demonstrou-se dificuldade de acesso. Aqui fica a questão, o estabelecimento do número de pacientes por equipe definido pela PNAB difere das recomendações internacionais, estaria aqui um fator primordial para dificuldade de acesso?

Entendemos que esta pesquisa cumpriu seus objetivos em acessar percepções sobre o acesso à APS de Curitiba e identificar barreiras. Levantamos também possibilidade que novos trabalhos com metodologias diferentes possam ajudar a dimensionar a questão do acesso. 


\section{APS em Revista}

Vol. 1, n. 3, p. 198/205 | Setembro/Dezembro - 2019

ISSN 2596-3317 - DOI 10.14295/aps.v1i3.39

Bizinelli, B. M.; Poli Neto, P.; Albuquerque, G. S. C.; Conde, R. E. S.; Scarin, F. C.

Como considerações para políticas de saúde, sugere-se não apenas adequação da quantidade de pessoas vinculadas por equipe, mas também a reorganização do processo de trabalho nas UBS, facilitando o acesso em até $48 \mathrm{~h}$. É necessário também avaliar o papel das UPA's no SUS.

\section{REFERÊNCIAS BIBLIOGRÁFICAS}

BARBOSA, S. P.; ELIZEU, T. S.; PENNA, C. M. M. Ótica dos profissionais sobre o acesso à atenção primária à saúde. Ciência \& Saúde Coletiva, v. 18, n. 8, p. 2347-2357, 2013.

BERCHET, C.; NADER, C. The organisation of out-ofhours primary care in OECD countries. OECD, 2016. Disponível em: < https://www.oecd-

ilibrary.org/docserver/5jlr3czbqw23-

en.pdf?expires $=1563746282 \&$ id=id\&accname $=$ guest\&checksum =D8098FC9DF5CEC0E80D31A F4AOF30C7E>. Acesso em: 21 de julho de 2019.

BRASIL. Ministério da Saúde. Portaria no 2.436, de 21 de setembro de 2017. Aprova a Política Nacional de Atenção Básica, estabelecendo a revisão de diretrizes para a organização da Atenção Básica, no âmbito do Sistema Único de Saúde (SUS). Diário Oficial da União, Brasília, DF, 21 set. 2017. Disponível em: <http://bvsms.saude.gov.br/bvs/saudelegis/g m/2017/prt2436_22_09_2017.html>. Acesso em: 17 de março de 2019.

CAMPOS, R. T. O. et al. Avaliação da qualidade do acesso na atenção primária de uma grande cidade brasileira na perspectiva dos usuários. Saúde Debate, v. 38 , n. especial, p. 252-64, 2014.

CARRAPATO, J. F. L. et al. Programa Mais Médicos: percepção dos usuários e dos profissionais do SUS. Em pauta, v. 14, n. 38, p. 280-293, 2016.

CASTRO, R. C. L. et al. Avaliação da qualidade da atenção primária pelos profissionais de saúde: comparação entre diferentes tipos de serviços. Cad. Saúde Pública, v. 28, n. 9, p. 1772-1784, 2012.
DISTRITO FEDERAL. Secretaria de Saúde do Distrito Federal. Manual de Acolhimento e Classificação de Risco. Brasília, DF, 2017. Disponível em:

http://www.saude.df.gov.br/wpconteudo/uploads/2017/10/MANUAL-DEACOLHIMENTO-E-

CLASSIFICA\%C3\%87\%C3\%830-DE-RISCO-DAREDE-SES-Web.pdf . Acesso em: 21 de julho de 2019.

FAORO, N. T. et al. Boletim de Informação e Monitoramento da Atenção Primária à Saúde da SMS Curitiba. Prefeitura Municipal de Curitiba. Secretaria Municipal da Saúde de Curitiba. 2015.

FONTANELLA, B. J. B. et al. Amostragem em pesquisas qualitativas: proposta de procedimentos para constatar saturação teórica. Cad. Saúde Pública, v. 2, n. 27, p. 389394, 2011.

FORD, J. A. et al. Weekend opening in primary care: analysis of the general practice patient survey. British Journal of General Practice, v. 65, n. 651, p. 792-798, 2018.

GODDARD, M. K. Quality in and equality of acess to healthcare services - Country report for England. England. European Commission, 2008. Disponível em: <https://ehma.org/wpContent/uploads/2016/08/healthquest_engla nd_en.pdf>. Acesso em: 15 de novembro de 2018.

GUSSO, G.; LOPES, J. M. C. Tratado de Medicina de Família e Comunidade. 1 a ed. Porto Alegre: Artmed Editora, 2012. p. 159-166.

MENDES, E. V. O Acesso à Atenção Primária à Saúde. Brasília: CONASS, p. 4-26, 2016.

MINAYO, M. C. S. O Desafio do Conhecimento. Pesquisa Qualitativa em Saúde. 14ạ ed. São Paulo: Hucitec, 2014, p. 303-308.

MURRAY, M.; BERWICK, D. M. Advanced acess: reducing waiting and delays in primary care. JAMA, v. 289, n. 9, p. 1035-1040, 2003.

O'DONNELL, P. et al. Exploring levers and barriers to accessing primary care for marginalised groups and identifying their priorities for primary care provision: a participatory learning and action research 
Vol. 1, n. 3, p. 198/205 | Setembro/Dezembro - 2019

ISSN 2596-3317 - DOI 10.14295/aps.v1i3.39

Bizinelli, B. M.; Poli Neto, P.; Albuquerque, G. S. C.; Conde, R. E. S.; Scarin, F. C.

study. International journal for equity in health, v. 197, n. 15, p. 1-16, 2016.

OLIVEIRA, A. E. F.; ARAUJO, F. L. S. M.; GARCIA, $P$. T. Redes de atenção à saúde: Rede de Atenção às Urgências e Emergências no Âmbito do Sistema Único de Saúde. 1a ed. São Luís: EDUFMA, 2018.

OLIVEIRA, S. N. et al. Unidade de pronto atendimento UPA $24 \mathrm{~h}$ : percepção da enfermagem. Texto Contexto Enferm, v. 24, n. 1, p. 238-244, 2015.

ORGANIZAÇÃO MUNDIAL DA SAÚDE. Conferência Internacional sobre Cuidados Primários em Saúde: relatório final. Alma-Ata, URSS, setembro de 1978.

PICOLI, R. P.; CAZOLA, L. H. O.; MAURER, N. M. J. S. Usuários de Classificação de Risco Azul em uma Unidade de Pronto Atendimento. Rev Cogitare Enfermagem, Curitiba, v. 21, n. 1, p. 17, 2016.

RAMOS, A. L.; HOFFMANN, F.; SPRECKELSEN. Waiting times in primary care depending on insurance scheme in Germany. BMC Health Services Research, v. 18, n. 191, p. 1-9, 2018.

ROCHA, A. S.; BOCCHI, S. C. M.; GODOY, M. F. Acesso aos cuidados primários da saúde: revisão integrativa. Physis Revista de Saúde Coletiva, v. 26, n. 1, p. 87-111, 2016.

SALISBURY, C. et al. The National Evaluation of NHS Walk-in Centres: final report. Bristol: University of Bristol, 2002. Disponível em: https://research-

information.bristol.ac.uk/files/9403884/WIC_E valuation_Report_Final.pdf.. Acesso em: 15 de novembro de 2018.

STARFIELD, B. Atenção primária: equilíbrio entre necessidades de saúde, serviços e tecnologia. Ed. Brasileira. Brasília: UNESCO, Ministério da Saúde, 2002.

SUBRAMANIAN, U. et al. Effect of advanced access scheduling on processes and intermediate outcomes of diabetes care and utilization. J Gen Interm Med, v. 24, n. 3, p. 327333, 2009.
TESSER, C. D. Por que é importante a prevenção quaternária na prevenção? Rev Saude Publica, v. 51, n. 116, p. 1-9, 2017.

TESSER, C. D.; NORMAN, A. H.; VIDAL, T. B Acesso na Atenção Primária à Saúde brasileira: situação, problemas e estratégias de superação. Saúde Debate, v. 42, n. 1, p. 361-78, 2018; 


\section{ABSTRACT}

Background: Scientific evidence indicates that a system based heavily on Primary Health Care promotes better health outcomes. In Brazil, Primary Health Care happens, in the public system, preferably in the Family Health Strategy model. The essential attributes are considered: access, longitudinality, coverage of services and coordination of care. This research aimed to explore the barriers of access to PHC in the view of users who attend a UPA (Emergency Care Unit).

Methods: Qualitative analysis based on semi-structured interviews with patients from a UPA of Curitiba, Paraná.

Results: The following barriers to access were found: distance from home, delayed response, understanding of system functioning, easy access to the Emergency Care Unit for non-urgent problems and few hours of operation of the UBS

Conclusions: There is a perception that the emergency unit has easier access than UBS, although it is not, in theory, the official gateway to the system.

Keywords: Primary Health Care, Access to Health Services, Health Assessment, Patients. 